in other studies-as tumor-selective delivery vehicles, and they demonstrate superior effects on survival. HB1.F3.C1 cells do not replicate in vivo, and they undergo apoptosis in noncancerous tissue. In addition, these cells migrate to subcutaneous xenografts of a variety of solid tumors and are found in the bone marrow only when tumor cells are present.

Original article Aboody KS et al. (2006) Development of a tumor-selective approach to treat metastatic cancer. PLoS ONE 1: e23

\section{Dichloroacetate decreases tumor growth and induces apoptosis in vitro and in vivo}

Aerobic glycolysis, high mitochondrial membrane potential $(\Delta \Psi \mathrm{m})$ and low expression of the potassium channel $\mathrm{K}_{\mathrm{v}} 1.5$ might confer apoptosis resistance on cancer cells. Dichloroacetate is a small metabolic modulator used in the treatment of lactic acidosis and inherited mitochondrial disorders. A study by Bonnet et al. has shown that dichloroacetate induces apoptosis and inhibits tumor growth in various cancer cells lines and animal models.

Incubation of human cancer cell lines with dichloroacetate restored the hyperpolarized $\Delta \Psi \mathrm{m}$ to the level of normal cells, decreased the lactic acid levels, and increased the intracellular $\mathrm{pH}$; however, dichloroacetate did not have any effect in healthy cells. Dichloroacetate activated $\mathrm{K}_{\mathrm{v}}$ channels in cancer cells, resulting in decreased intracellular potassium, and increased $\mathrm{K}_{\mathrm{v}} 1.5$ expression via a mechanism dependent on nuclear factor of activated T lymphocyte 1 (NFAT). The authors found that $\mathrm{K}_{\mathrm{v}} 1.5$ expression was inversely correlated with the tumor grade in samples from patients with non-small-cell lung cancer. Dichloroacetate induced apoptosis in cancer cells through two mechanisms: depolarization that activated mitochondria-dependent apoptosis; and upregulation of $\mathrm{K}_{\mathrm{v}} 1.5$ channels. Nude athymic rats that received dichloroacetate prior or after implantation of human cancer cells showed a marked decrease in tumor growth compared with untreated animals, confirming the in vitro data.

Although these results need validation in clinical trials, this study suggests that dichloroacetate might be a promising agent for the treatment of a wide spectrum of human malignancies.

Original article Bonnet S et al. (2007) A mitochondria- $\mathrm{K}^{+}$ channel axis is suppressed in cancer and its normalization promotes apoptosis and inhibits cancer growth. Cancer Cell 11: $37-51$

\section{PALB2 is a novel breast cancer susceptibility gene}

Biallelic mutations in PALB2, a gene implicated in the nuclear localization and stability of BRCA2, have been shown by Rahman and coworkers to cause Fanconi anemia with phenotype similar to that caused by BRCA2 biallelic mutations. The same group has also shown that monoallelic truncating PALB2 mutations also confer susceptibility to breast cancer.

The study included 923 individuals with familial breast cancer, negative for mutations in BRCA1 and BRCA2, and 1,084 controls. Of the 923 individuals with familial breast cancer, 10 were found to carry truncating PALB2 mutations, compared with none of the controls (1.1\% and $0 \%$, respectively, $P=0.0004)$. The median ages at diagnosis for individuals with and without PALB2 mutations were 46 and 49 years, respectively $(P=0.24)$, suggesting that breast cancer risks associated with PALB2 mutations might be age-dependent. Individuals with PALB2 mutations had a 2.3-fold higher risk of breast cancer than did those without a mutation $(P=0.0025)$. The relative risks of breast cancer for PALB2 mutation carriers under 50 years and over 50 years of age were 3.0 and 1.9 , respectively. With the exception of one individual, who developed melanoma in addition to breast cancer, carriers of PALB2 mutations developed no malignancies other than breast cancer. The increase in breast cancer risk associated with $P A L B 2$ is lower than that conferred by BRCA2 mutations.

This study is the first to suggest a role for PALB2 in breast cancer predisposition. The reasons for the increased risk associated with PALB2 mutations are unknown and further studies of the gene are required.

Original article Rahman N et al. (2007) PALB2, which encodes a BRCA2-interacting protein, is a breast cancer susceptibility gene. Nat Genet 39: 165-167 